\title{
Controlled Sign Reversal of Electroresistance in Oxide Tunnel Junctions by Electrochemical-Ferroelectric Coupling
}

\author{
D. Hernandez-Martin $\odot,{ }^{1}$ F. Gallego, ${ }^{1,2}$ J. Tornos, ${ }^{1,2}$ V. Rouco, ${ }^{1}$ J. I. Beltran, ${ }^{1,3}$ C. Munuera $\oplus^{2,4}$ \\ D. Sanchez-Manzano, ${ }^{1}$ M. Cabero, ${ }^{1}$ F. Cuellar $\odot,{ }^{1}$ D. Arias, ${ }^{1, *}$ G. Sanchez-Santolino $\odot,{ }^{1,2,3}$ F. J. Mompean, ${ }^{2,4}$ \\ M. Garcia-Hernandez, ${ }^{2,4}$ A. Rivera-Calzada, ${ }^{1,4}$ S. J. Pennycook,${ }^{5}$ M. Varela, ${ }^{1,3}$ M. C. Muñoz, ${ }^{4,6}$ \\ Z. Sefrioui, ${ }^{1,4,7}$ C. Leon, ${ }^{1,4,7}$ and J. Santamaria ${ }^{1,4,7}$ \\ ${ }^{1}$ GFMC, Universidad Complutense de Madrid, 28040 Madrid, Spain \\ ${ }^{2} 2$ D-Foundry Group, Instituto de Ciencia de Materiales de Madrid ICMM-CSIC, 28049 Madrid, Spain \\ ${ }^{3}$ Instituto Pluridisciplinar, Universidad Complutense de Madrid, 28040 Madrid, Spain \\ ${ }^{4}$ Unidad Asociada UCM/CSIC, Laboratorio de Heteroestructuras con Aplicación en Spintrónica, 28049 Madrid, Spain \\ ${ }^{5}$ Department of Materials Science \& Engineering, National University of Singapore, Singapore 117575 \\ ${ }^{6}$ Instituto de Ciencia de Materiales de Madrid ICMM-CSIC, 28049 Madrid, Spain \\ ${ }^{7}$ GFMC, Instituto de Magnetismo Aplicado, Universidad Complutense de Madrid, 28040 Madrid, Spain
}

(Received 24 July 2019; accepted 24 November 2020; published 30 December 2020)

\begin{abstract}
The persistence of ferroelectricity in ultrathin layers relies critically on screening or compensation of polarization charges which otherwise destabilize the ferroelectric state. At surfaces, charged defects play a crucial role in the screening mechanism triggering novel mixed electrochemical-ferroelectric states. At interfaces, however, the coupling between ferroelectric and electrochemical states has remained unexplored. Here, we make use of the dynamic formation of the oxygen vacancy profile in the nanometerthick barrier of a ferroelectric tunnel junction to demonstrate the interplay between electrochemical and ferroelectric degrees of freedom at an oxide interface. We fabricate ferroelectric tunnel junctions with a $\mathrm{La}_{0.7} \mathrm{Sr}_{0.3} \mathrm{MnO}_{3}$ bottom electrode and $\mathrm{BaTiO}_{3}$ ferroelectric barrier. We use poling strategies to promote the generation and transport of oxygen vacancies at the metallic top electrode. Generated oxygen vacancies control the stability of the ferroelectric polarization and modify its coercive fields. The ferroelectric polarization, in turn, controls the ionization of oxygen vacancies well above the limits of thermodynamic equilibrium, triggering the build up of a Schottky barrier at the interface which can be turned on and off with ferroelectric switching. This interplay between electronic and electrochemical degrees of freedom yields very large values of the electroresistance (more than $10^{6} \%$ at low temperatures) and enables a controlled switching between clockwise and counterclockwise switching modes in the same junction (and consequently, a change of the sign of the electroresistance). The strong coupling found between electrochemical and electronic degrees of freedom sheds light on the growing debate between resistive and ferroelectric switching in ferroelectric tunnel junctions, and moreover, can be the source of novel concepts in memory devices and neuromorphic computing.
\end{abstract}

DOI: 10.1103/PhysRevLett.125.266802

The study of the novel electronic states appearing in (both) structurally and chemically sharp interfaces between correlated oxides has become one of the paradigms of an emerging field [1]. Oxygen vacancies, however, are defects difficult to detect and in many cases also to avoid in these oxides [2], and may have drastic effects on materials properties through their associated strain and doping fields [3]. In oxide nanostructures, defect formation energy may be substantially reduced, and as a result, oxygen vacancies can be generated under the action of external electric fields and accumulate at boundaries enabling the modification of their electrochemical state [4-6]. Recently, coupling between electrochemical and ferroelectric states has been found at surfaces of ultrathin ferroelectric layers [7]. In this Letter, we make use of the generation and transport of oxygen vacancies to modify the electric response of the ultrathin barrier of a ferroelectric tunnel junction and demonstrate the role of ferroelectric-electrochemical coupling at interfaces in the mechanism of tunnel electroresistance of ferroelectric tunnel junctions.

The discovery that ferroelectricity can survive in ultrathin layers if polarization charges can be effectively screened at metallic boundaries [8] has opened the way to tunnel junctions with ferroelectric barriers. These devices have been the focus of much interest in recent years due to the possibility of modulating the tunneling resistance by the orientation (up or down) of the ferroelectric polarization in what is called tunneling electroresistance [8]. Notably, a giant electroresistance has been theoretically predicted $[8,9]$ and experimentally observed 
[10-18] for ferroelectric capacitors with metal electrodes with different screening lengths. There is a growing debate in the literature on the origin of the large hysteretic changes in the tunneling conductance driven by the application of moderate electric fields. Some works have shown that electroresistance is solely determined by the domain structure of the ferroelectric (defining multiple memresistance states) [10]. Typically, this approach relies on the use of narrow (100 ns) pulse sequences to switch ferroelectric polarization and avoid the drift of oxygen vacancies. Other authors have shown large hysteretic resistance changes in ferroelectric tunnel junctions resulting from the drift of oxygen vacancies $[19,20]$. Furthermore, samples produced with high intentional concentration of oxygen vacancies display reversed polarity (resistive) switching to the ferroelectric switching found in similar samples with low vacancy concentration [21]. Both types of switchings have even been found in the same sample but appearing independently at different voltage ranges [22,23]. There is an interesting open question on the possible interplay between both phenomena as it could enable the electrochemical control of ferroelectric polarization and be the source of novel electroresistance effects resulting from the coupling between ferroelectric polarization and charged defects at interfaces and their possible role in novel device concepts [7].

Here we explore the interplay between ferroelectric electroresistance and the electrochemical defect generation by using a radically new approach. By applying (continuous) dc fields to promote electrochemical reactions in a ferroelectric ultrathin layer, we are able to control the generation and transport of oxygen vacancies across it and explore their effect on the switching of the ferroelectric polarization. We demonstrate a strong coupling between ferroelectric polarization and ionized oxygen vacancies. The manipulation of the oxygen vacancies profile at the interfaces of the ferroelectric barrier modifies the screening mechanism of ferroelectric polarization, and vice versa, ferroelectric polarization triggers a strong modulation of the concentration of ionized vacancies at the oxide or ferroelectric interface. We use this behavior to demonstrate a new functionality in a tunnel junction device: a controlled and reversible activation or deactivation of a strong memristive response in the device by simply applying a large enough dc bias of different polarity.

We have grown ferroelectric $\mathrm{BaTiO}_{3}$ (BTO) on $\mathrm{Sr}$ doped La manganite $\mathrm{La}_{0.7} \mathrm{Sr}_{0.3} \mathrm{MnO}_{3}$ (LSMO) epitaxially deposited onto (001) $\mathrm{SrTiO}_{3}$ (STO) substrates using a high (pure) oxygen pressure sputtering technique. These LSMO/BTO interfaces are sharp both structurally and chemically as shown by scanning transmission electron microscopy (Nion UltraSTEM 200) high angle annular dark field (HAADF) imaging [see Fig. 1(a)] combined with electron energy-loss spectroscopy (EELS) elemental maps. Analyzing the fine structure of the $L_{2,3}$ edges, we
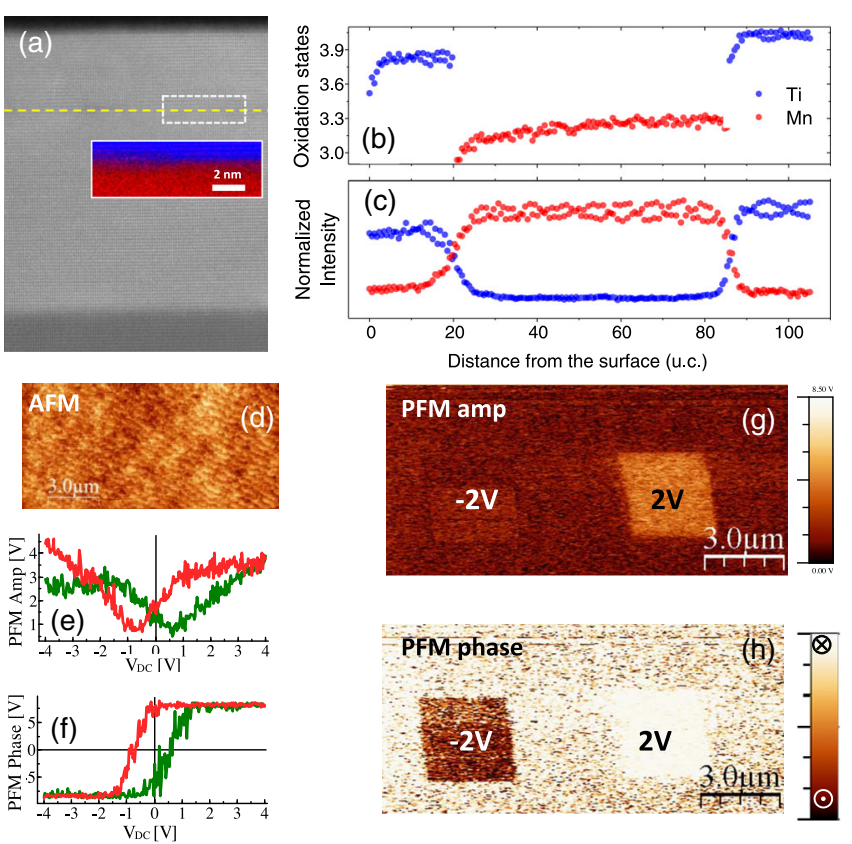

FIG. 1. Interface structure. (a) Atomic resolution HAADF image of the $\mathrm{SrTiO}_{3}(100) / / 25 \mathrm{~nm} \mathrm{La}_{0.7} \mathrm{Sra}_{0.3} \mathrm{MnO}_{3}(\mathrm{LSMO}) / 10 \mathrm{~nm}$ $\mathrm{BaTiO}_{3}$ heterostructure down the [110] direction. The inset shows an EELS Ti $L_{2,3}$ (blue), La $M_{4,5}$ (red) integrated intensity map acquired from the marked area at the LSMO/BTO interface. (b) Ti and $\mathrm{Mn}$ oxidation states and (c) normalized integrated intensity profiles along the STO//LSMO/BTO heterostructure. (d) AFM image showing atomically flat surfaces displaying STO surface terraces. Piezoresponse [amplitude (e) and phase (f)] hysteresis loop measured on a selected location of the sample. Amplitude (g) and phase (h) PFM images showing that stable polarization states can be written with the PFM tip using small voltages. Panels (g) and (h) show ferroelectric domains generated applying $\pm 2 \mathrm{~V}$ (as labeled) tip voltages.

quantified the Ti oxidation state using a multiple linear least-squares fit to two reference spectra for bulk $\mathrm{LaTiO}_{3}$ $\left(\mathrm{Ti}^{+3}\right)$ and $\mathrm{BaTiO}_{3}\left(\mathrm{Ti}^{+4}\right)$ [24]. We found a significant reduction of the Ti oxidation state in the BTO layer, which takes values of 3.85 (instead of the nominal 4) probably resulting from the nucleation of oxygen vacancies at the surface [see Fig. 1(b)]. The imaging of light atoms in bright field images supplied a direct measurement of the ferroelectric polarization (see Supplemental Material [25], Fig. S1) [24], which took values close to the bulk polarization of BTO and showed preferred downorientation, probably due to the accumulation of oxygen vacancies at the surface $[34,35]$. Atomic force microscopy imaging shows that the surface topography after the growth of the LSMO and the BTO top layer reproduces the oneunit-cell-thick STO substrate terraces evidencing a 2D growth [see Fig. 1(d)]. Piezoelectric force microscopy (PFM) using amplitude and phase contrast indicates a ferroelectric ground state and the possibility to "write" up or down polarization states using a few volts tip bias 

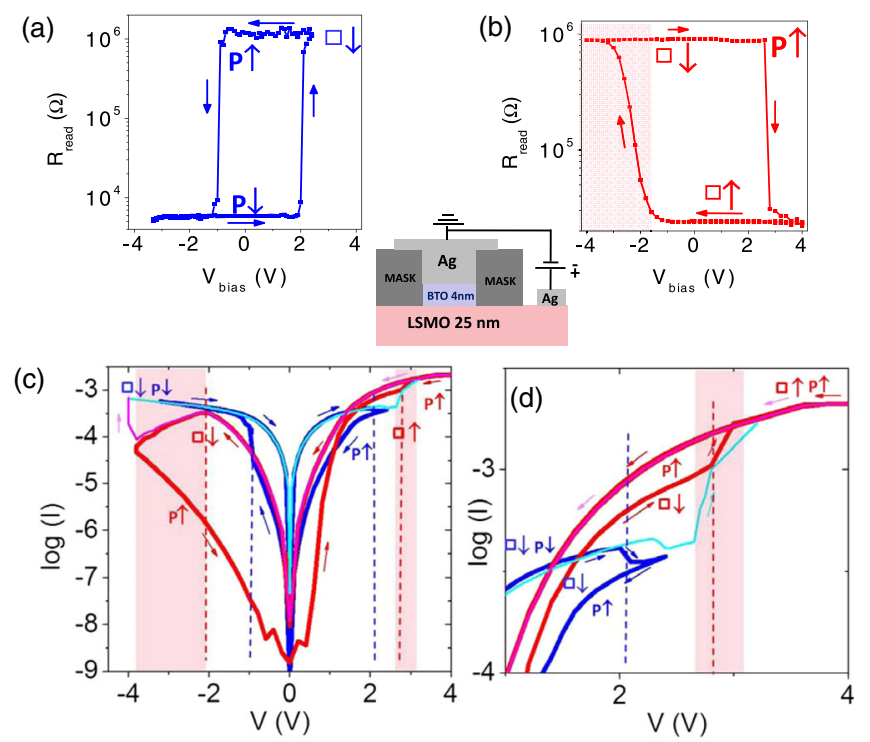

FIG. 2. Ferroelectric and oxygen vacancy resistance loops. Electroresistance loops of an Ag/BTO/LSMO bilayer with initial state written with electric field pointing down (negative voltage) (a), and with electric fields pointing up (positive voltage) (b). After applying each $V_{\text {bias }}$, junction resistance is measured under $V_{\text {read }}=10 \mathrm{mV}$ (a) and $V_{\text {read }}=0.7 \mathrm{~V}$ (b). The different resistance states are identified according to the orientation up $(P \uparrow)$ or down $(P \downarrow)$ of the ferroelectric polarization and to the location up ( $\square \uparrow$ ) or down ( $\square \downarrow$ ) of oxygen vacancies (i.e., oxygen vacancies pushed by electric field against the top or the bottom BTO interface). (c) $I-V$ curves of an Ag/BTO/LSMO sample measured at $100 \mathrm{~K}$. Blue (red) curves are measured after initial resistance states written in electric fields pointing down (up). Larger amplitude sweeps with negative starting voltage (cyan curve) and with positive starting voltage (light magenta curve) switch between the two families of curves (blue and red). (d) Enlarged view of the $I-V$ curves at positive voltages to observe resistance switches.

[see Figs. 1(e)-1(h) showing ferroelectric domains generated applying $\pm 2 \mathrm{~V}$ tip voltages).

$\mathrm{La}_{0.7} \mathrm{Sr}_{0.3} \mathrm{MnO}_{3}(25 \mathrm{~nm}) / \mathrm{BaTiO}_{3}(4 \mathrm{~nm}) / \operatorname{Metal}(\mathrm{Ag})$ micron-size pillars were fabricated by using conventional optical lithography techniques and ion milling (an example is displayed in the inset of Fig. S2 in the Supplemental Material [25]). With such a small barrier thickness, electron transport is governed by tunneling through the oxide barrier as previously shown in symmetric $\mathrm{La}_{0.7} \mathrm{Sr}_{0.3} \mathrm{MnO}_{3}(10 \mathrm{~nm}) / \mathrm{BaTiO}_{3}(4 \mathrm{~nm}) /$ $\mathrm{La}_{0.7} \mathrm{Sr}_{0.3} \mathrm{MnO}_{3}(25 \mathrm{~nm})$ magnetic tunnel junctions [24]. $\mathrm{Ag}$ and LSMO have very similar work functions (4.7 and $4.8 \mathrm{eV}$, respectively), and thus, both are expected to build up similar contact barriers given by the energy difference between the work function of the metal and the electron affinity of the BTO insulator $(3.8 \mathrm{eV})$. The selection of the $\mathrm{Ag}$ electrode serves the purpose of generating oxygen vacancies [36]. Ag has an excellent oxygen diffusivity and solubility, and (as Pt or $\mathrm{Au}$ ) it is catalytically active in the process of oxygen dissociation yielding accelerated oxygen exchange $[37,38]$. It is well established that under applied electric fields, oxygen vacancy generation and reduction of BTO will proceed as described by the following reaction: $\mathrm{O}_{\mathrm{O}} \rightarrow \mathrm{V}{ }^{\prime} \mathrm{o}+1 / 2 \mathrm{O}_{2}+2 \mathrm{e}^{-}$where $\mathrm{V}$ " $\mathrm{o}$ denotes a doubly ionized oxygen vacancy in the relative charge (Kröger-Vink) notation. Charged oxygen vacancies generated under the $\mathrm{Ag}$ electrode are driven by the electric field to the bottom LSMO where they accumulate. Electrons to compensate charge neutrality are supplied by the LSMO electrode. Single ionized oxygen vacancies have a doping effect associated with a donor level $0.4 \mathrm{eV}$ below the conduction band edge [39], and their accumulation forms a virtual cathode at the LSMO interface with modified conducting properties.

We have measured hysteretic electroresistance on the tunnel junctions in the temperature range $20-100 \mathrm{~K}$ by recording the resistance $\left(R_{\text {read }}\right)$ at low read voltages $\left(V_{\text {read }}\right)$ after voltage steps $\left(V_{\text {write }}\right)$ following the loops of Figs. 2(a) and 2(b). The top Ag electrode was grounded (see sketch in Fig. 2), so that positive (negative) voltages correspond to electric fields pointing up (down). Prior to the electroresistance measurement, two distinct initial resistance states were written by strong 5-8 $\mathrm{V}$ negative or positive voltages. In the state written with the negative voltage, with slightly lower resistance, the ferroelectric polarization points down to the bottom electrode $(P \downarrow)$ and eventual oxygen vacancies accumulate at the LSMO interface state $(\square \downarrow)$ (blue curves in Fig. 2). On the contrary, in the initial state written with positive voltage, polarization points up $(P \uparrow)$ to the Ag electrode, and vacancies are pushed against the Ag interface ( $\square \uparrow$ ) (red curves in Fig. 2).

We first discuss the data at $100 \mathrm{~K}$ as representative of the whole dataset since, as shown in the Supplemental Material [25], Sec. 2, there is a weak temperature dependence due to the tunneling-dominated transport across the ultrathin ferroelectric barrier. Changing the polarity of the voltage used to write the initial state triggers a sign change of the electroresistance defined in terms of the (positive) saturation fields $E_{\text {sat }}$ as $\left[R\left(E_{\text {sat }}\right)-R\left(-E_{\text {sat }}\right) / R\left(E_{\text {sat }}\right)\right]$. Initial states written by electric fields pointing down (up) produced a positive (negative) electroresistance.

The positive electroresistance loops measured at $10 \mathrm{mV}$ (with initial states written with negative voltages) switch steeply at voltages comparable to those found in the PFM experiments and can be naturally assigned according to the interface polarization model of the giant electroresistance of ferroelectric capacitors [9] to the inversion of the ferroelectric polarization [see Fig. 2(a)]. The resistance increase at positive voltages (switch to ferroelectric polarization pointing up) is expected within this model due to the increase of the average barrier height when polarization points away from the LSMO electrode with much longer Thomas Fermi screening length than silver. The switching of the ferroelectric polarization produced large values of the 
electroresistance in the range $10^{4} \%-10^{5} \%$, as expected from the large interface asymmetry due to the different contacts [9]. Similar samples with (nonferroelectric) $\mathrm{SrTiO}_{3}$ barriers showed very different switching behavior, which provides further support of the ferroelectric origin of these switches and also allow ruling out that they are due to the formation of $\mathrm{Ag}$ filaments known to occur in oxide insulators at (and above) room temperature [40] (see the Supplemental Material [25], Sec. 3). The negative electroresistance loops [red curves in Fig. 2(b)] with larger coercive voltages were obtained in the high resistance state written initially with electric fields pointing up and were measured at a (read) voltage level of $0.7 \mathrm{~V}$ necessary to achieve a measurable (low) resistance value (see below). The sign change of the electroresistance indicates a different underlying mechanism (other than the inversion of the ferroelectric polarization). We will argue below that it is due to the switching of oxygen vacancies. Hysteretic $I-V$ curves measured in the same temperature and (write) voltage range as electroresistance loops showed also a markedly different behavior for the two initial resistance states. The red (blue) curves in Fig. 2(c) corresponding to initial resistance states written by electric fields pointing up (down) are clockwise (anticlockwise), which again indicates two different mechanisms. The initial states written with electric fields pointing down (blue curves) produce switching between two tunnelinglike $I-V$ curves (with parabolic voltage-dependent conductance) which occur at the same coercive fields as the ferroelectric electroresistance loops indicating that hysteresis is due to the switching of the ferroelectric polarization [for clarity, Fig. 2(d) shows an enlarged view to better identify the switches at positive voltages]. In fact, analysis of $I-V$ curves at low voltages with the Brinkman model for a trapezoidal tunnel barrier [41] (see Fig. 3) evidences a strong modulation of the tunneling barrier by the switching of the ferroelectric polarization. The barrier height determined by the distance from the Fermi level to the edge of the BTO conduction band changed between 0.12 and $0.23 \mathrm{eV}$ for down and up polarization, respectively (see Fig. 3 and sketches).

On the other hand, initial electric fields pointing up [red curves in Fig. 2(c)] trigger a dramatic change of the shape of the tunnel barrier. $I-V$ curves between positive voltages down to $-2 \mathrm{~V}$ are tunnel-like with larger barrier heights close to $0.35 \mathrm{eV}$ [as obtained from the analysis with the Brinkman model; see Fig. 3(c)]. Sweeps toward more negative voltages $(V<-2 \mathrm{~V})$ evidence a gradual crossover from tunnel- into Schottky-like $I-V$ curves denounced by a strong suppression of the current at negative voltages. See also Fig. 2(c) reproduced in linear scale as Supplemental Material [25], Fig. S2, which clearly evidences the Schottky regime. Fits of $I-V$ curves to the Schottky model yields a $0.7 \mathrm{eV}$ barrier [see Fig. 3(d)] building up at the bottom interface, since it is reverse biased for negative voltages. The large barrier height implies a
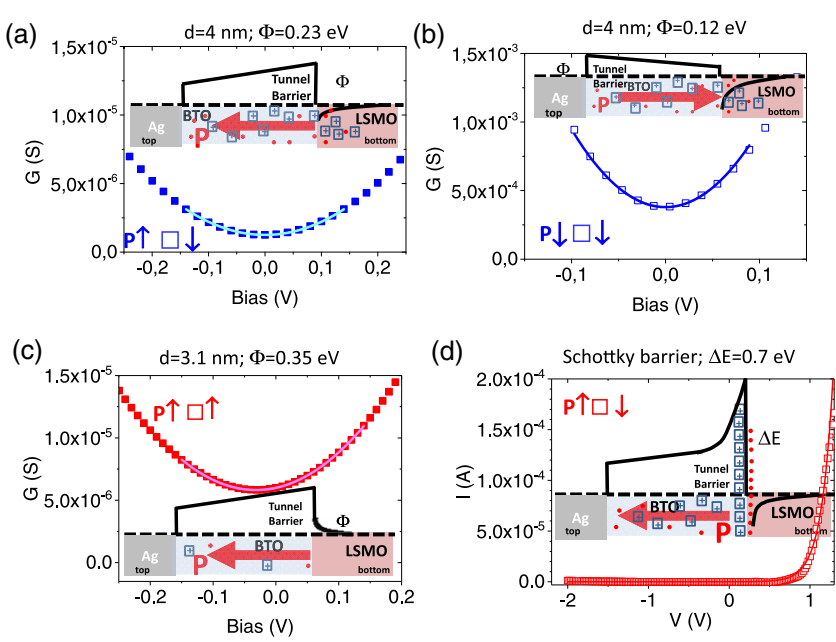

FIG. 3. Resistive and ferroelectric switching of Ag/BTO/LSMO bilayers. $I-V$ curves of an Ag/BTO/LSMO sample measured at $100 \mathrm{~K}$ analyzed with the Brinkmann-Dynes-Rowell model (Ref. [41]) in the tunneling regime [panels (a)-(c)] and with a Schottky model [panel (d)]. Upper and lower sketches in panels (a) and (b) illustrate the modulation of the tunnel barrier due to the asymmetric screening at the interfaces according to the interface polarization model [9]. Sketches in panels (c) and (d) illustrate the crossover from tunnel regime (c) to Schottky regime (d). Voltage in panel (d) is swept from negative to positive.

strong ionization of the oxygen vacancies (located $0.4 \mathrm{eV}$ below conduction band edge) as depicted in the sketch to Fig. 3(d). Moreover, the very gradual nature of the resistance switch associated with the build up of the Schottky barrier [marked with a pink shaded rectangle in Fig. 2(c)] is a first indication that it is due to the electrochemical generation of oxygen vacancies and not to the reversal of the ferroelectric polarization which typically occurs with sharper coercive fields.

Theoretical investigation using $a b$ initio simulations support the modulation of the shape of the tunnel barrier by the ionization of the oxygen vacancies. Electronic structure calculations were performed in $\mathrm{Ag} / \mathrm{BTO} / \mathrm{LSMO}$ isolated heterostructures with oxygen vacancies introduced in the $\mathrm{TiO}_{2}$ plane either at the top $(\mathrm{Ag} / \mathrm{BTO})$ interface or at the bottom (BTO/LSMO) interface to model the two initial states ( $\square \uparrow$ and $\square \downarrow$ ) written with electric fields pointing up ( $\square \uparrow$ ) or down $(\square \downarrow)$. The barrier heights obtained from the electronic structure calculations compare satisfactorily with those obtained from the tunneling conductance curves of Fig. 3 (see Supplemental Material [25], Fig. S7), which supports the interpretation that the modulations of the tunnel barrier are caused by the interplay between ferroelectric polarization and the generation and ionization of oxygen vacancies.

A direct confirmation of this scenario is obtained from the measurements of low voltage capacitance. Frequencydependent capacitance and conductance were measured (HP 4285 LCR meter) with low voltage $(20 \mathrm{mV})$ excitation 

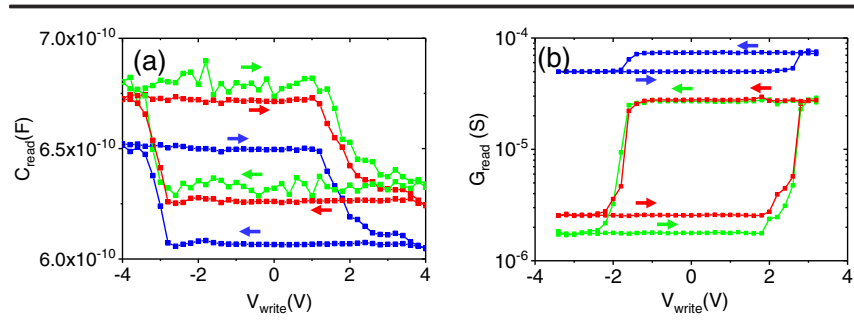

FIG. 4. Hysteretic capacitance in the Schottky regime. Low voltage $(20 \mathrm{mV})$ capacitance (a) and conductance (b) loops recorded at $100 \mathrm{~K}$ at various frequencies: $1 \mathrm{kHz}$ (green symbols), $10 \mathrm{kHz}$ (red symbols), and $100 \mathrm{kHz}$ (blue symbols).

ac signals using an impedance analyzer. Capacitance and conductance displayed a hysteretic behavior when voltage was swept in a hysteresis loop sequence (see Fig. 4) after applying a positive voltage to write the initial resistance state. (Negative initial voltages produced nonhysteretic capacitance plots.) Counterclockwise conductance loops were obtained, consistent with the clockwise resistance plots obtained in this regime. The amplitude of the conductance loops decreased with increasing frequency until eventually hysteresis disappeared. See the Supplemental Material [25], Sec. 5 (Figs. S8 and S9). The capacitance increase $\left(\Delta C=5 \times 10^{-11} \mathrm{~F}\right)$ with respect to the $6 \times 10^{-10} \mathrm{~F}$ value of the parasitic (parallel) capacitance of the devices observed at the negative switching voltage is strong evidence of a charge process associated with the generation and ionization of vacancies at the LSMO/BTO interface. Notice that contrary to conductance, capacitance excess is not frequency dependent in our frequency range, which evidences that charge accumulation and tunneling conductance represent two separate (series) dielectric processes.

The picture now clearly emerges that while the positive electroresistance is due to the switching of the ferroelectric polarization, the negative electroresistance is due to the build up of a Schottky barrier due to the accumulation of ionized oxygen vacancies in the space charge region. In fact, both switching processes occur sequentially as shown by the light blue and magenta $I-V$ curves of Fig. 3(c) measured from both initial states in an extended voltage range. But interestingly, as we discuss below, both processes are coupled.

Charged vacancies generated at the negative switching voltage accumulate at the $\mathrm{BTO} / \mathrm{LSMO}$ interface to compensate the negative polarization charges (of the up-polarization state) building an interface dipole (Schottky barrier). The size of the interface dipole can be estimated from the capacitance hysteresis as $\Delta \mathrm{C}=(\Delta \mathrm{Q} / \Delta \phi)$, which relates the increment of the capacitance $\left(\Delta \mathrm{C}=5 \times 10^{-11} F\right)$ to the barrier height $\Delta \phi$ and the charge $\Delta Q$ stored at one side of the interface. Using the value of $\Delta \phi=0.7 \mathrm{~V}$ obtained for the barrier height, $\Delta \mathrm{Q}=3.5 \times 10^{-11} \mathrm{C}$ can be obtained. Dividing by the area of the device $\left(A=136 \mu \mathrm{m}^{2}\right)$, a value of $26 \mu \mathrm{C} \mathrm{cm}^{-2}$ results in very good agreement with the value of BTO bulk polarization. This evidences an electrochemicalferroelectric (ferroionic) coupling [7] triggered by the screening of the polarization charges by the ionized oxygen vacancies which (1) controls the ionization of oxygen vacancies and (2) strongly stabilizes ferroelectric polarization.

The generation of oxygen vacancies in this polarization up state $(P \uparrow, \square \downarrow)$ triggers their extraordinary high level of ionization giving rise to the Schottky barrier responsible for the very large resistance difference [see Fig. 2(b)] between state $(P \uparrow, \square \downarrow)$ and state $(P \uparrow, \square \uparrow)$ where oxygen vacancies are pushed toward the top contact by the positive field where they partially annihilate. This yields a nearly infinite oxygen vacancy electroresistance at low voltage, as shown by the resistance difference between the red open and solid symbols in Fig. S3(e) of the Supplemental Material [25]. Physically, this scenario can be understood in the framework of models where ferroelectric polarization is compensated or screened at interfacial Schottky barriers building at interfaces due to the accumulation of defects [42-46]. Interestingly, the gradual voltage-controlled vacancy generation process delicately controls the height of the Schottky barrier allowing for a multiplicity of memresistance states which can be reached in minorvoltage loops (see Supplemental Material [25], Fig. S10). Notice that barrier formation does not happen when ferroelectric polarization reverses to point in the up direction in the presence of previously existing oxygen vacancies $[P \uparrow, \square \downarrow$; the high resistance state in the blue curves of Fig. 2(a)], where their low level of ionization is set by thermodynamic equilibrium (a donor level $0.4 \mathrm{eV}$ below the conduction band edge which will be weakly ionized at the low temperatures of the experiment).

Conversely, resulting from accumulation of oxygen vacancies at the bottom interface, the polarization up state now switches at a much higher voltage of nearly $-4 \mathrm{~V}$ instead of the $-1 \mathrm{~V}$ coercive voltage [see magenta curve in Fig. 2(c)] demonstrating that the compensation of polarization charges by ionized oxygen vacancies has a stabilizing effect on the ferroelectricity up to relatively high fields, above which, coupled oxygen vacancies and polarization switch from up to down and the Schottky barrier collapses as the result of the neutralization of oxygen vacancies. The stabilization of the polarization up by oxygen vacancies is also supported by the density-functional-theory calculations as discussed in the Supplemental Material [25], Sec. 4.

In summary, we have shown the coupling between electric-field-driven oxygen vacancy generation and ferroelectric polarization switching at an artificial ferroelectric interface of a tunnel junction device. We have demonstrated that the screening of polarization by charged oxygen vacancies increases the ionization of the donor level associated with oxygen vacancies well above the level 
set by thermodynamic equilibrium. This ferroelectricdriven ionization builds up an interface dipole which controls tunnel transport yielding a nearly infinite electroresistance and memristive response. Moreover, the significance of the coupling in a single device between ferroelectricity and electrochemical responses involving ion motion, and the interplay of this coupling with electronic processes, goes far beyond current applications of memristors, so far restricted to mimic synapses, and may trace interesting new avenues for emulation of the activity of whole neurons.

All relevant data present in this publication can be accessed at Ref. [47].

The authors acknowledge received funding from the project Quantox of QuantERA ERA-NET Cofund in Quantum Technologies (Grant Agreement No. 731473) implemented within the European Union's Horizon 2020 Programme. Work supported by Spanish MINECO through Grants No. MAT2014-52405-C02, No. MAT2017-87134C02, No. MAT2015-066888-C3-1-3-R, and No. RTI2018097895-B-C41 (-C43), Grant No. Santander-UCM PR87/ 19-22603, and computational support from Grant No. FI-2019-1-0012. J. S. thanks for the scholarship program Alembert funded by the IDEX Paris-Saclay, Grant No. ANR-11-IDEX-0003-02.

* On leave from Universidad del Quindio, 630000 Armenia, Colombia.

[1] H. Boschker and J. Mannhart, Annu. Rev. Condens. Matter Phys. 8, 145 (2017).

[2] J. N. Eckstein, Nat. Mater. 6, 473 (2007).

[3] S. V. Kalinin and N. A. Spaldin, Science 341, 858 (2013).

[4] C. Leighton, Nat. Mater. 18, 13 (2019).

[5] D. A. Gilbert, A. J. Grutter, P. D. Murray, R. V. Chopdekar, A. M. Kane, A. L. Ionin, M. S. Lee, S. R. Spurgeon, B. J. Kirby, B. B. Maranville, A. T. N'Diaye, A. Mehta, E. Arenholz, K. Liu, Y. Takamura, and J. A. Borchers, Phys. Rev. Mater. 2, 104402 (2018).

[6] P. D. Murray, D. A. Gilbert, A. J. Grutter, B. J. Kirby, D. Hernández-Maldonado, M. Varela, Z. E. Brubaker, W. L. N. C. Liyanage, R. V. Chopdekar, V. Taufour, R. J. Zieve, J. R. Jeffries, E. Arenholz, Y. Takamura, J. A. Borchers, and K. Liu, ACS Appl. Mater. Interfaces 12, 4741 (2020).

[7] S. M. Yang, A. N. Morozovska, R. Kumar, E. A. Eliseev, Y. Cao, L. Mazet, N. Balke, S. Jesse, R. K. Vasudevan, C. Dubourdieu, and S. V. Kalinin, Nat. Phys. 13, 812 (2017).

[8] J. P. Velev, C. G. Duan, J. D. Burton, A. Smogunov, M. K. Niranjan, E. Tosatti, S. S. Jaswal, and E. Y. Tsymbal, Nano Lett. 9, 427 (2009).

[9] M. Y. Zhuravlev, R. F. Sabirianov, S. S. Jaswal, and E. Y. Tsymbal, Phys. Rev. Lett. 94, 246802 (2005).

[10] V. Garcia, S. Fusil, K. Bouzehouane, S. Enouz-Vedrenne, N. D. Mathur, A. Barthélémy, and M. Bibes, Nature (London) 460, 81 (2009).
[11] P. Maksymovych, S. Jesse, P. Yu, R. Ramesh, A. P. Baddorf, and S. V. Kalininl, Science 324, 1421 (2009).

[12] A. Gruverman, D. Wu, H. Lu, Y. Wang, H. W. Jang, C. M. Folkman, M. Y. Zhuravlev, D. Felker, M. Rzchowski, C. B. Eom, and E. Y. Tsymbal, Nano Lett. 9, 3539 (2009).

[13] D. Pantel, S. Goetze, D. Hesse, and M. Alexe, ACS Nano 5, 6032 (2011).

[14] D. Pantel, H. Lu, S. Goetze, P. Werner, D. Jik Kim, A. Gruverman, D. Hesse, and M. Alexe, Appl. Phys. Lett. 100, 232902 (2012).

[15] D. Pantel, S. Goetze, D. Hesse, and M. Alexe, Nat. Mater. 11, 289 (2012).

[16] L. Jiang, W. S. Choi, H. Jeen, S. Dong, Y. Kim, M. G. Han, Y. Zhu, S. V. Kalinin, E. Dagotto, T. Egami, and H. N. Lee, Nano Lett. 13, 5837 (2013).

[17] Z. Wen, C. Li, D. Wu, A. Li, and N. Ming, Nat. Mater. 12, 617 (2013).

[18] X. Liu, J. D. Burton, and E. Y. Tsymbal, Phys. Rev. Lett. 116, 209902 (2016).

[19] Q. H. Qin, L. Äkäslompolo, N. Tuomisto, L. Yao, S. Majumdar, J. Vijayakumar, A. Casiraghi, S. Inkinen, B. Chen, A. Zugarramurdi, M. Puska, and S. van Dijken, Adv. Mater. 28, 6852 (2016).

[20] J. Li, N. Li, C. Ge, H. Huang, Y. Sun, P. Gao, M. He, C. Wang, G. Yang, and K. Jin, iScience 16, 368 (2019).

[21] M. Li, J. Zhou, X. Jing, M. Zeng, S. Wu, J. Gao, Z. Zhang, X. Gao, X. Lu, J. M. Liu, and M. Alexe, Adv. Electron. Mater. 1, 1500069 (2015).

[22] R. Soni, A. Petraru, P. Meuffels, O. Vavra, M. Ziegler, S. K. Kim, D. S. Jeong, N. A. Pertsev, and H. Kohlstedt, Nat. Commun. 5, 5414 (2014).

[23] W. Lü, C. Li, L. Zheng, J. Xiao, W. Lin, Q. Li, X. R. Wang, Z. Huang, S. Zeng, K. Han, W. Zhou, K. Zeng, J. Chen, A. Ariando, W. Cao, and T. Venkatesan, Adv. Mater. 29, 1606165 (2017).

[24] G. Sanchez-Santolino, J. Tornos, D. Hernandez-Martin, J. I. Beltran, C. Munuera, M. Cabero, A. Perez-Muñoz, J. Ricote, F. Mompean, M. Garcia-Hernandez, Z. Sefrioui, C. Leon, S. J. Pennycook, M. C. Muńoz, M. Varela, and J. Santamaria, Nat. Nanotechnol. 12, 655 (2017).

[25] See Supplemental Material at http://link.aps.org/ supplemental/10.1103/PhysRevLett.125.266802 for control experiments and temperature dependence of the electroresistance, which includes Refs. [26-33]].

[26] J. Tornos et al., Phys. Rev. Lett. 122, 037601 (2019).

[27] Z. Sefrioui, D. Arias, M. Varela, J. E. Villegas, M. A. López de la Torre, C. León, G. D. Loos, and J. Santamaría, Phys. Rev. B 60, 15423 (1999).

[28] F. A. Cuellar, Y. H. Liu, J. Salafranca, N. Nemes, E. Iborra, G. Sanchez-Santolino, M. Varela, M. G. Hernandez, J. W. Freeland, M. Zhernenkov, M. R. Fitzsimmons, S. Okamoto, S. J. Pennycook, M. Bibes, A. Barthélémy, S. G. E. Te Velthuis, Z. Sefrioui, C. Leon, and J. Santamaria, Nat. Commun. 5, 4215 (2014).

[29] M. Varela, M. P. Oxley, W. Luo, J. Tao, M. Watanabe, A. R. Lupini, S. T. Pantelides, and S. J. Pennycook, Phys. Rev. B 79, 085117 (2009).

[30] E. Mikheev, B. D. Hoskins, D. B. Strukov, and S. Stemmer, Nat. Commun. 5, 3990 (2014). 
[31] S. D. Findlay, N. Shibata, H. Sawada, E. Okunishi, Y. Kondo, and Y. Ikuhara, Ultramicroscopy 110, 903 (2010).

[32] K. Tsunoda, Y. Fukuzumi, J. R. Jameson, Z. Wang, P. B. Griffin, and Y. Nishi, Appl. Phys. Lett. 90, 113501 (2007).

[33] X. B. Yan, K. Li, J. Yin, Y. D. Xia, H. X. Guo, L. Chen, and Z. G. Liu, Electrochem. Solid-State Lett. 13, H87 (2010).

[34] A. K. Tagantsev, C. Z. Pawlaczyk, K. Brooks, and N. Setter, Integr. Ferroelectr. 4, 1 (1994).

[35] A. K. Tagantsev and G. Gerra, J. Appl. Phys. 100, 051607 (2006).

[36] S. Q. Liu, N. J. Wu, and A. Ignatiev, Appl. Phys. Lett. 76, 2749 (2000).

[37] M. Leonhardt, R. A. De Souza, J. Claus, and J. Maier, J. Electrochem. Soc. 149, J19 (2002).

[38] N. J. Simrick, J. A. Kilner, and A. Atkinson, Thin Solid Films 520, 2855 (2012).
[39] Y. Xiao, V. B. Shenoy, and K. Bhattacharya, Phys. Rev. Lett. 95, 247603 (2005).

[40] C. Yoon, J. H. Lee, S. Lee, J. H. Jeon, J. T. Jang, D. H. Kim, Y. H. Kim, and B. H. Park, Nano Lett. 17, 1949 (2017).

[41] W. F. Brinkman, R. C. Dynes, and J. M. Rowell, J. Appl. Phys. 41, 1915 (1970).

[42] P. W. M. Blom, R. M. Wolf, J. F. M. Cillessen, and M. P. C. M. Krijn, Phys. Rev. Lett. 73, 2107 (1994).

[43] L. Pintilie and M. Alexe, J. Appl. Phys. 98, 124103 (2005).

[44] L. Pintilie, I. Boerasu, M. J. M. Gomes, T. Zhao, R. Ramesh, and M. Alexe, J. Appl. Phys. 98, 124104 (2005).

[45] S. Farokhipoor and B. Noheda, APL Mater. 2, 056102 (2014).

[46] H. T. Yi, T. Choi, S. G. Choi, Y. S. Oh, and S. W. Cheong, Adv. Mater. 23, 3403 (2011).

[47] J. Santamaria (2020), https://doi.org/10.5281/zenodo.3615617. 